\title{
AVALIAÇÃO DA SATISFAÇÃO EM UM HOSPITAL FILANTRÓPICO: comparativo entre usuários dos serviços público e privado
}

\author{
Monica de Mônico Magalhães \\ Hospital Madre Regina Protmann (ACSC), Santa Teresa-ES, Brasil
}

Salus Vitae

Introdução

No âmbito da utilização do controle de qualidade para obtenção de vantagem competitiva entre as instituições de saúde, este trabalho objetiva comparar a satisfação percebida entre os usuários dos serviços públicos e privados atendidos numa mesma instituição filantrópica. Para isto, utilizou-se de uma ferramenta de avaliação padronizada de cuidados hospitalares a partir da perspectiva do cliente, desenvolvida pelo Centers of Medicare and Medicaid Services (EUA) e de domínio público chamada HCAHPS (Hospital Consumer Assessment of Healthcare Providers and Systems), com tradução para a língua portuguesa já existente.

\section{Procedimento Experimental}

Trabalho de pesquisa quantitativo de caráter descritivo com registro, analise e interpretação dos dados da população de usuários do setor privado e público internados em um hospital filantrópico do Espírito Santo. A amostra foi composta de cinquenta usuários, sendo vinte e cinco do setor público e vinte e cinco do setor privado. Classificada como intencional, tipo não-probabilística de conveniência. A coleta de dados foi realizada utilizando-se um questionário estruturado com 30 (trinta) questões, chamado ferramenta HCAHPS (Hospital Consumer Assessment of Heathcare Providers and Systems, criado pela Agência NorteAmericana de Pesquisa e Qualidade em Saúde, de domínio público e versão em língua portuguesa. Os dados obtidos para cada tópico avaliado foram tabulados seguindo o mesmo modelo de apresentação de resultados utilizado para divulgação da pesquisa HCAHPS nos Estados Unidos. Este modelo classifica as respostas em três vertentes de importância: a "top-box" onde se concentra a maior pontuação para o quesito, a "middle-box" que reúne a pontuação intermediária e a "bottombox", que concentra a menor pontuação.

\section{Resultados}

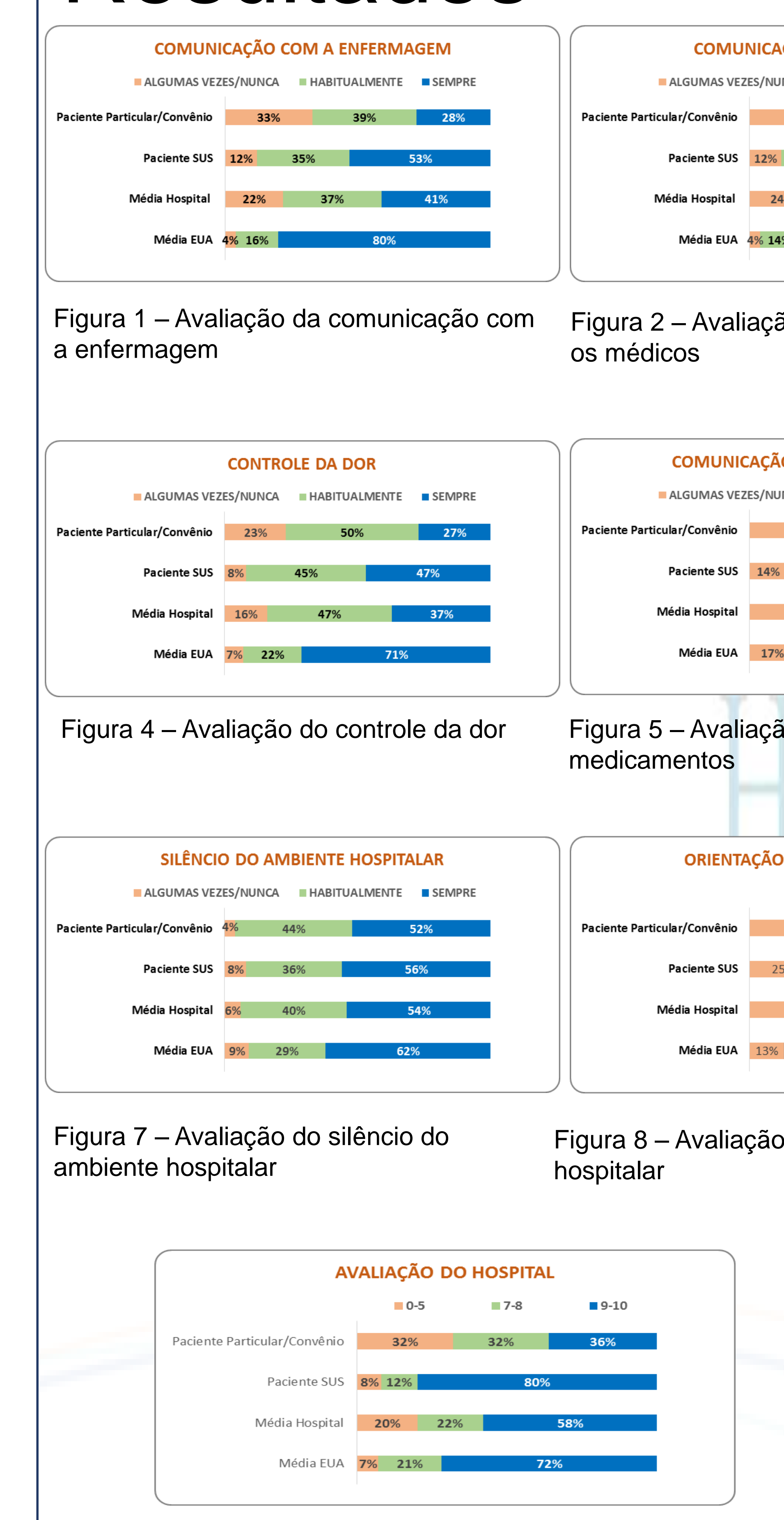

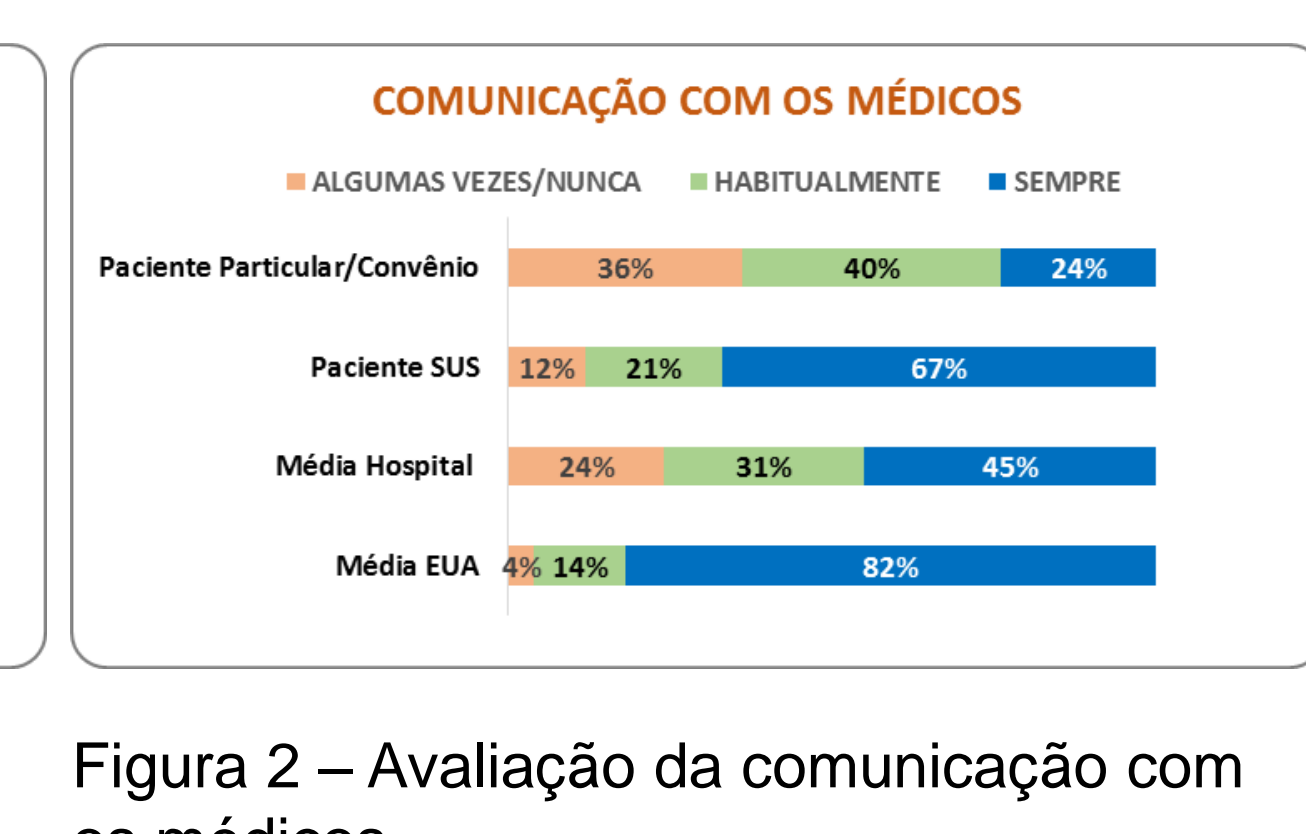
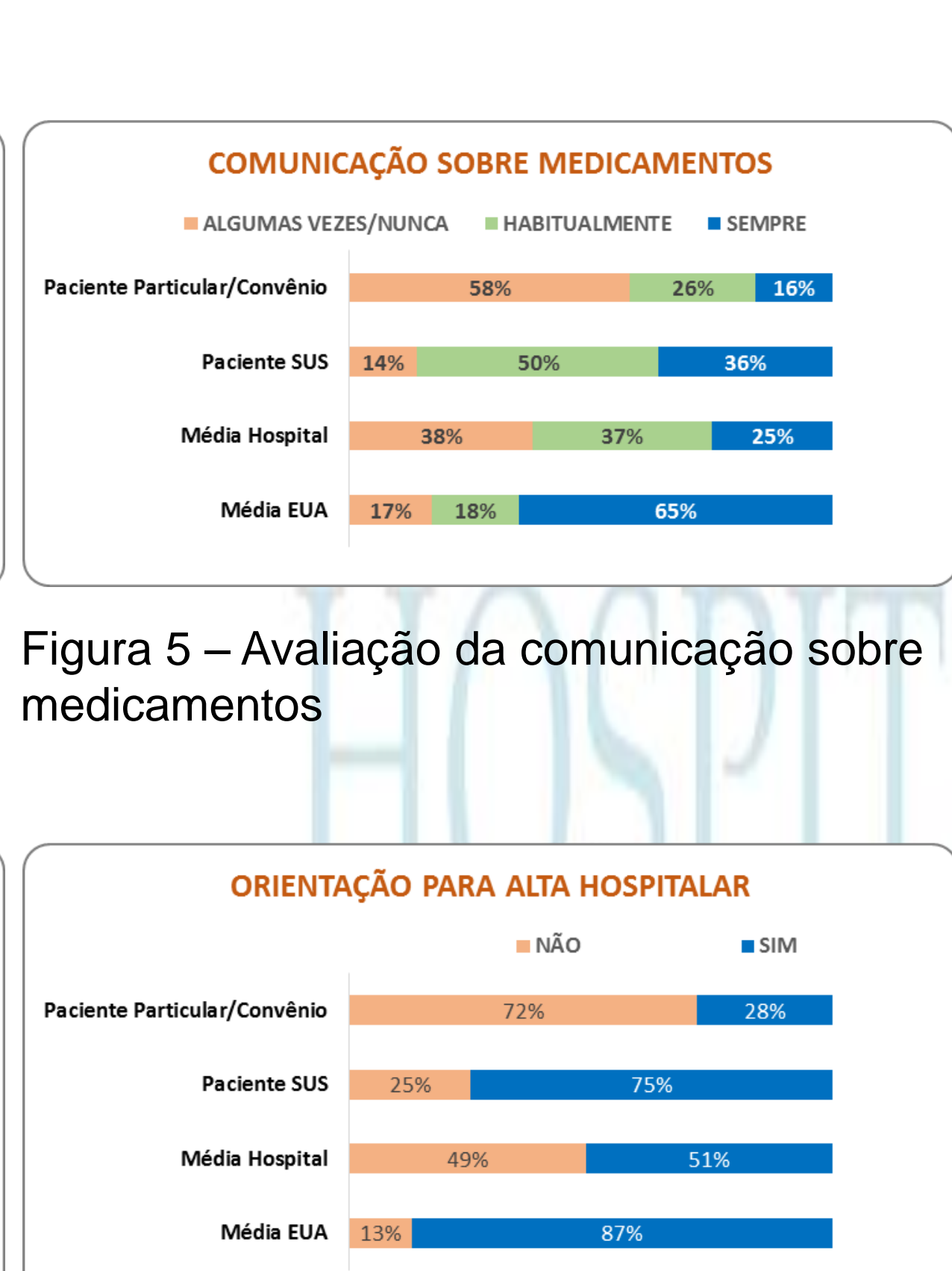

Figura 8 - Avaliação da orientação para alta hospitalar

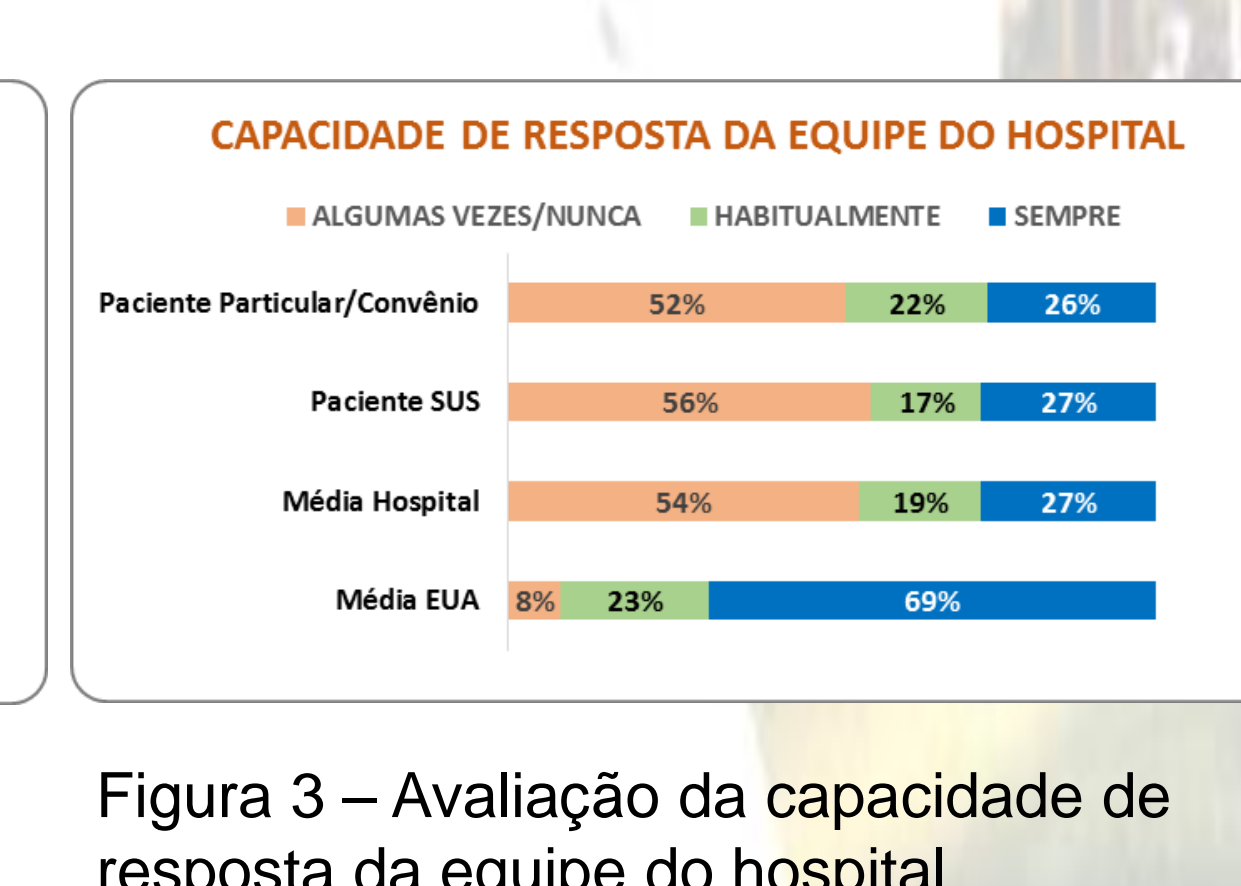

Figura 3 - Avaliação da capacidade
resposta da equipe do hospital

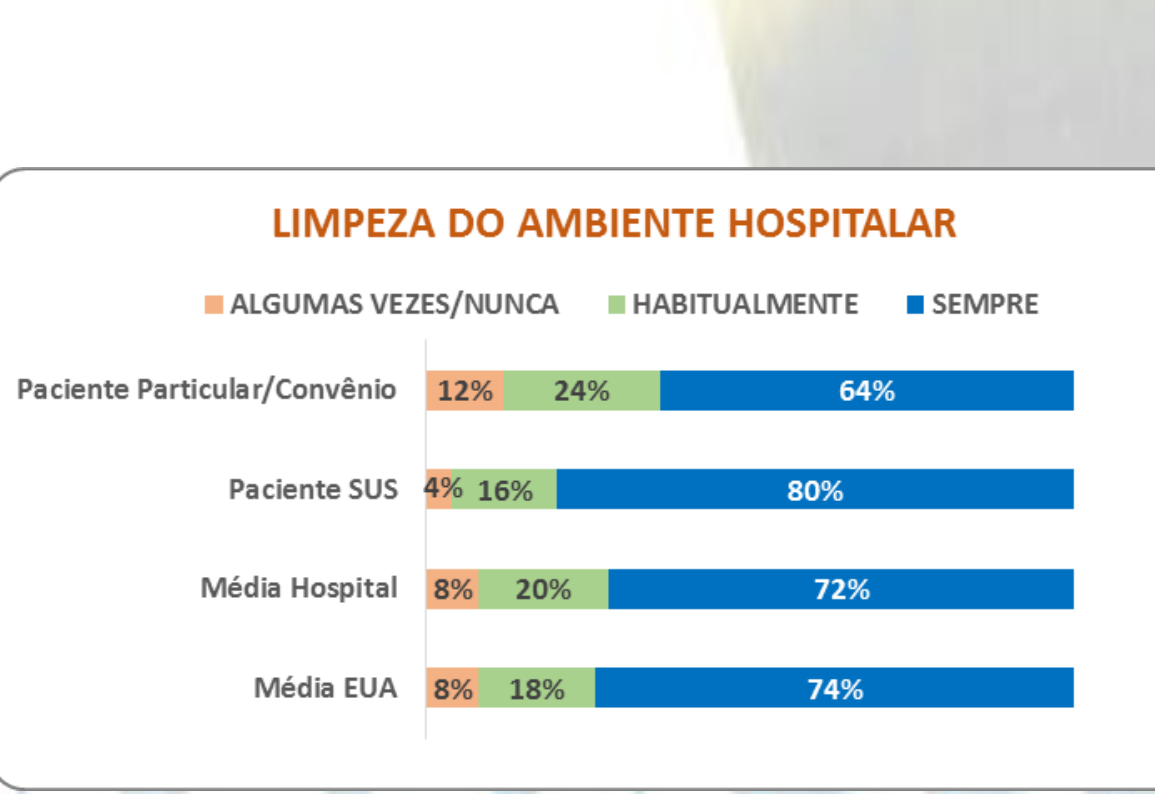

Figura 6 - Avaliação da limpeza do ambiente hospitalar

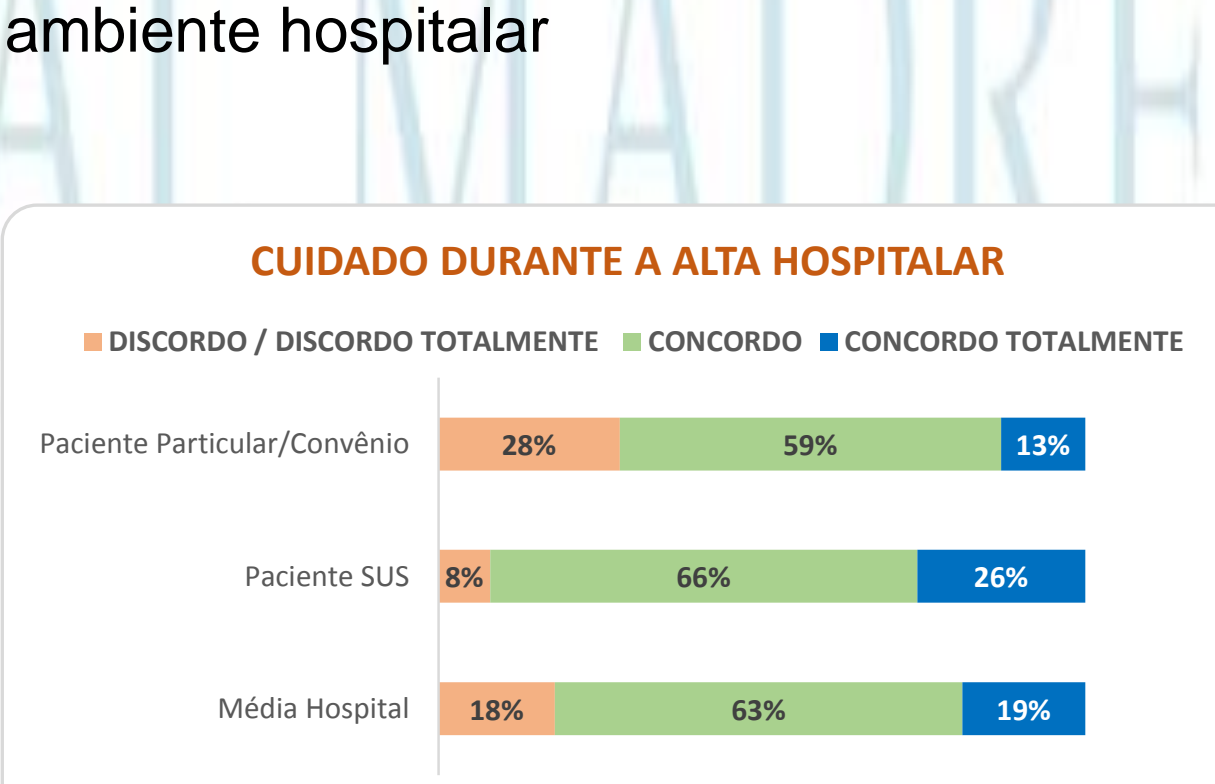

Figura 9 - Avaliação parcial do cuidado com o paciente durante a alta hospitalar

\section{Conclusão}

Em última análise, podemos concluir que a utilização da ferramenta HCAHPS é de fácil aplicação, podendo oferecer dados para reflexão sobre a melhoria dos processos de cuidados centrados no paciente, ampliando a visão humanística das instituições através da qualidade percebida pelos usuários. Também é possível afirmar que há uma diferença de percepção de qualidade dentro das dimensões estudadas entre os usuários desta instituição e os usuários dos hospitais americanos, não podendo ser afirmado se isto se deve a uma real diferença de cuidados ou se por aspectos sociais, econômicos e culturais das duas populações, que não foram levados em conta neste trabalho. As diferenças entre as avaliações dos usuários da rede SUS e da rede privada na mesma instituição deveu-se principalmente aos aspectos intangíveis da avaliação, o que torna desafiador a proposição de ações, uma vez que o resultado é dependente da preferência do usuário.

Para futuros trabalhos será necessário ampliar a utilização da ferramenta utilizada para outras instituições com o intuito de termos parâmetros regionais e nacionais de comparabilidade e talvez o desenvolvimento de um desenho de pesquisa que consiga avaliar o nível de exigência das diferentes populações de usuários, além de uma extensão no tempo de aplicação da ferramenta para que se dissolva o viés da transversalidade da amostra.

\section{Referências}

BAKI, B.; PEKER, I. An Integrated Evaluation Model for Service Quality of Hospitals: A Case Study From Turkey. Journal of Multiple-Valued Logic \& Soft Computing, v. 24, n. 5, P. 453-475, 2015.

CENTERS FOR MEDICARE \& MEDICAID SERVICES. Hospital Consumer Assessment of Healthcare Provider and Systems. Disponível em: <http://www.hcahpsonline.org>. Acesso em: 20 de out. 2016.

CHASSIN, M. R.; LOEB, J. M.; SCHMALTZ, S. P.; WACHTER, R. M. Accountability mea
quality improvement. New England Journal of Medicine, v. 363, n. 7, p. 683-688, 2010

CHOI, K. S., CHO, W. H.; LEE, S.; LEE, H.; KIM, C. The relationships among quality, value, satisfaction and behavioral intention in health care provider choice: A South Korean study. Journal of Business Research, v. 57, n. 8, p. 913-921, 2004.

CINAROGLU, S. Complexity in healthcare management: Why does Drucker describe healthcare organizations as a doubleheaded monster? International Journal of Healthcare Management, v. 9, n. 1, p. 11-17, 2016

CRONIN JR, J. J.; BRADY, M.K.; HULT, G.; TOMAS M. Assessing the Effects of Quality, Value, and Customer Satisfaction on Consumer Behavioral Intentions in Service Environments. Journal of Retailing, v. 76, n. 2, p. 193-218, 2000.

DONABEDIAN, A. The quality of care: how can it be assessed?. Jama, v. 260, n. 12, p. 1743-1748, 1988.

PLSEK, P. E.; GREENHALGH, T. The challenge of complexity in health care. British Medical Journal, v. 323, n. 7313, p. 625, 2001.

PRICE, R.A.; ELLIOTT, M.N.; ZASLAVSKY, A.M.; HAYS, R.D.; LEHRMANN, W.G,; RYBOSLKI, L.; EDGNAN-LEVITAN, L.; CLEARY, P.D. Examining the Role of Patiente Experience Surveys in Measuring Health Care Quality. Medical Care Research and Review, v. 71, n.5, p. 522-524, 2014

ROCHA, L.R.M.; VEIGA, D. F.; OLIVEIRA, P.R.; SONG, E. H.; FERREIRA, L. M. Health service quality scale: Braziliam Portuguese translation, reliability and validity. BMC Health Services Research, v. 13, n. 1, p. 24-29, 2013.

TONTINI, G.; WALTER, S. A.; COSTA, M.; FREGA, J.R. Satisfação com serviços hospitalares como fator estratégico de gestão: uma abordagem não-linear. Gestão \& Planejamento-G\&P, v. 14, n. 3, p. 580-593, 2013. ZEITHAML, V. A.; BERRY, L. L.; PARASURAMAN, A. The behavioral consequences of service quality. The
Marketing, v. 60, n. 4, p. 31-46, 1996 . 